\title{
FRECUENCIA DE INFECCIONES RESPIRATORIAS AGUDAS EN MENORES DE 5 AÑOS, CENTRO DE SALUD RIO BLANCO, 2017
}

\author{
FREQUENCY OF ACUTE RESPIRATORY INFECTIONS IN UNDER 5 YEARS \\ OLD, RIO BLANCO HEALTH CENTER, 2017
}

Brian Iván Zurita Céspedes ${ }^{1, a}$, Boris Inturias Imaca', David Laura Vargas ${ }^{1}$

\section{RESUMEN}

Introducción: Las infecciones respiratorias agudas (IRAS) se definen como el conjunto de enfermedades del aparato respiratorio causadas por microorganismos virales, bacterianos u otros, caracterizados síntomas respiratorios y son consideradas la mayor causa de morbimortalidad infantil en países en vías de desarrollo. Objetivo: Describir la frecuencia de IRAS en menores de 5 años atendidos en el Centro de Salud Rio Blanco de enero a diciembre del año 2017. Materiales y Métodos: Se trata de un estudio descriptivo, de corte transversal. El universo son todos los menores de 5 años que acudieron al Centro de Salud Río Blanco del 2017, siendo en total 3 5I0. Resultados: se identificó un $31 \%$ de frecuencia de IRAS, un $44 \%$ de faringoamigdalitis como diagnostico principal, leve predominio no significativo en el sexo femenino, mayor frecuencia en edades mayores de 2 años y mayor frecuencia en invierno. Conclusiones: se evidencia una alta frecuencia de IRAS en la población estudiada con características similares a las estimadas en la literatura para las condiciones del medio.

\section{ABSTRACT}

Introduction: Acute respiratory infections (IRAS) are defined as the set of diseases of the respiratory system caused by viral, bacterial or other microorganisms, characterized by respiratory symptoms and are considered the greatest cause of infant morbidity and mortality in developing countries. Objective: To describe the frequency of IRAS in children under 5 years attended at the Rio Blanco Health Center from January to December of the year 2017. Materials and Methods: This is a descriptive, cross-sectional study. The universe is all those under 5 years of age who attended the Río Blanco Health Center in 2017, with a total of 3510 . Results: $31 \%$ of IRAS frequency was identified, $44 \%$ of pharyngotonsillitis as the main diagnosis, slight predominance of significant in the female sex, higher frequency in ages older than 2 years and higher frequency in winter. Conclusions: a high frequency of IRAS is evident in the studied population with characteristics similar to those estimated in the literature for the environmental conditions.

\section{INTRODUCCIÓN}

as infecciones respiratorias agudas (IRAS)

son un conjunto de enfermedades del sistema respiratorio causadas por agentes virales, bacterianos u otros microorganismos que ocasionan sintomatología respiratoria'. Son consideradas la mayor causa de morbimortalidad infantil en países en vías de desarrollo, seguidas por las enfermedades diarreicas agudas (EDAS) ${ }^{1-3}$. Las IRAS afectan cualquier porción del tracto respiratorio, siendo su variabilidad clínica dependiente de la porción afectada ${ }^{4}$. No obstante, varias de ellas pueden manifestar síntomas generales. Las IRAS pueden ocurrir en cualquier periodo del año, pero incrementan considerablemente en invierno con un leve predominio no significativo en el sexo masculino ${ }^{2}$. Al ser enfermedades infecciosas, transmisibles, su frecuencia está relacionada con el modo de vida de la población y está estrechamente ligada a los factores biológicos individuales, como a las condiciones sociales y sanitarias; es así que influyen para su desarrollo: el bajo peso al nacer, la falta de inmunización, la mal nutrición, el uso previo de antibióticos, la contaminación ambiental, el hacinamiento, la falta de educación en los cuidadores y el escaso acceso a la atención médica ${ }^{1,2}$. Las IRAS representan hasta el $40 \%$ de las consultas y el $30 \%$ de las hospitalizaciones en todo el mundo. Dentro de las complicaciones de las IRAS se ha reportado que la neumonía con sobre infección bacteriana
${ }^{1}$ M.D.- Médico Cirujano. a Diplomado en Tutoria para la investigacion en Salud y Diplomado en emergencias.

Correspondencia a:

Nombre: Brian Iván Zurita Céspedes

Correo electrónico:

brian_2501@hotmail.com

Telf. y celular: +59172277767

ORCID: https://orcid.org/00000001-8949-7217.

Palabras clave: Enfermedades respiratorias, faringitis, centros de salud, estaciones del año.

Keywords: Respiratory Tract Diseases, Pharyngitis, Health Centers, Seasons.

Procedencia y arbitraje: no comisionado, sometido a arbitraje externo.

Recibido para publicación: 28 de mayo de 2020 Aceptado para publicación: 4 de noviembre de 2020

Citar como:

Zurita Céspedes BI, Inturias Imaca B, Laura Vargas D. Frecuencia de infecciones respiratorias agudas en menores de 5 años, centro de salud río blanco, 2017. Rev Cient Cienc Med 2020; 23(2): 201-206 
es responsable de hasta 156 millones de casos a nivel mundial, cuya mortalidad alcanza el $20 \%$ del total de casos en países en vías de desarrollo mientras que solo representa el 3\% en países desarrollados. Datos de América Latina indican que la mortalidad por neumonía alcanza al $14 \%$ de todas las defunciones, cifra 10 veces superior a la de países desarrollados ${ }^{5}$.

Según datos del Instituto Nacional de Estadística (INE), en Bolivia, 41,6\% de los menores de 5 años estarían afectados por IRAS con una mayor prevalencia en niñas $(42,4 \%)$ en relación a niños (40,9\%). De acuerdo con el INE se observa que a nivel regional en Cochabamba el $34.3 \%$ de los menores de 5 años entre niños y niñas presentan IRAS (datos publicados tras la Encuesta de Hogares llevada a cabo por el INE en 2017) ${ }^{6}$. Se ha estimado que cada niño desarrollaría entre 5 a 9 infecciones del aparato respiratorio cada año, sobre todo en menores de 2 años en los que se evidencia mayor compromiso del tracto respiratorio superior. Si bien dichas infecciones suelen tener baja letalidad representan una importante fuente para alguna complicación posterior, por ejemplo, una neumonia ${ }^{2}$. El Centro de Salud San Martin de Porres de Rio Blanco es un centro de atención en salud de primer nivel ubicado en localidad que lleva por nombre Rio Blanco del municipio Entre Ríos, provincia Carrasco, en Cochabamba, Bolivia. Mismo que brinda atención médica en sus servicios de consulta externa y emergencias las 24 horas del día, los 365 días del año. Gran parte de la población que solicita sus servicios vive en condiciones deficientes de higiene y sanidad, no cuentan con agua potable y en muchos casos tampoco con servicio de alcantarillado. Se ha percibido gran afluencia de menores de 5 años en los servicios de consulta externa y emergencias de dicho centro de salud, cuyos cuidadores referían como motivo de consulta síntomas respiratorios. Es así, que se ha planteado el siguiente objetivo de investigación: describir la frecuencia de IRAS en menores de 5 años atendidos en el Centro de Salud Rio Blanco de enero a diciembre del año 2017.

\section{MATERIALES Y MÉTODOS}

El presente es un estudio de tipo Descriptivo, de corte transversal, retrospectivo.
Las fuentes de información fueron los libros de registro de Atención Integral del Niño Menor de Cinco Años, disponibles en el Centro de Salud Rio Blanco (C.S. Rio Blanco).

El universo del estudio fueron todos los menores de 5 años atendidos en el C.S. Rio Blanco entre los meses de enero y diciembre del año 2017, siendo en total 3 510. Dentro los criterios de inclusión se ha considerado menores de 5 años que hayan sido atendidos en el tiempo mencionado, en los que producto de la valoración clínica se determinó el diagnóstico de IRA; los criterios de exclusión contemplan registros con diagnósticos diferentes a IRAS, además de pacientes con registros de edad y genero ausentes, con ello se obtuvo la unidad de análisis con una cifra similar (3 510) y se trabajó con la misma, por lo que no se obtuvo muestra.

Las variables identificadas fueron: diagnóstico clínico, género, edad, y estaciones del año.

Los instrumentos de recolección de datos fueron una Planilla de Registro de información y Planilla de Recolección de Resultados.

Procedimiento.- se realizó la revisión sistemática de los libros de Atención Integral del Niño Menor de Cinco Años del año 2017, con la búsqueda y selección de pacientes menores de 5 años con diagnóstico de IRAS, los hallazgos se registraron en una planilla de registro de información, posteriormente en una planilla de obtención de datos, y finalmente fueron trasladados a una planilla de obtención de resultados tras su respectivo análisis estadístico.

Las consideraciones éticas de esta investigación se delinean en base a los lineamientos de la declaración de Helsinki, actualizada en la Asamblea General de la Asociación Médica Mundial, en Fortaleza Brasil, Octubre 2013; dentro de ello obtuvo la debida autorización de las autoridades del C.S. Rio Blanco para realizar el estudio y se ha mantenido en total anonimato los datos identificativos y resultados individuales de la población estudiada.

Los datos obtenidos fueron tabulados y analizados con el programa Microsoft Office Excel $2010^{\circledR}$ bajo estadística Descriptiva con obtención de valores absolutos $\left(\mathrm{N}^{\circ}\right)$ y relativos (\%).

\section{RESULTADOS}


En total recibieron atención 3510 menores de 5 años con diferentes patologías, de los cuales $31,23 \%$ (N: 1096) tienen algún tipo de IRA (Ver

\section{Grafica 1).}

Según el diagnóstico, la Faringoamigdalitis Aguda encabeza las IRAS con 44\% (n: 485) del total seguido del resfrío común con $37 \%$ ( $\mathrm{n}$ : 404), y la Otitis Media Aguda en último lugar con $1 \%$ (n: 14) del total (Ver Grafica 2).

Según el género se observó un 49\% (n: 535) de frecuencia en varones y un 51\% (n: 561) de frecuencia en mujeres (Ver Tabla 1), y según la edad niños y niñas con 3 años cumplidos reportaron frecuencias de 46,6\% (n: 156), en cambio pacientes con 1 año cumplido registraron solo $25,8 \%$ (n: 298 ) dentro su grupo etáreo, siendo el menor en comparación con los otros grupos (Ver Grafica 3).

Finalmente, se evidenció una frecuencia de $31,66 \%$ (n: 347) de IRAS en invierno seguida de las restantes estaciones del año, estas últimas con similares proporciones (Ver Grafica 4)

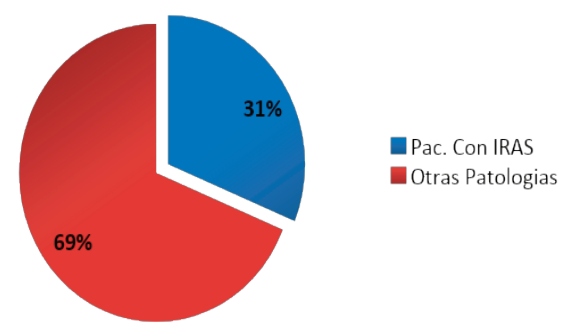

Gráfica 1. Frecuencia de IRAS en menores de 5 años, C.S. Río Blanco, 2017. Se evidencia un $31 \%$ de frecuencia de IRAS en todos los menores de 5 años ( $N=3$ 510). Fuente: PROPIA (Autor cubierto por doble ciego)

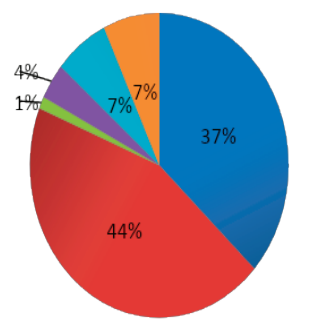

¿esfrio Común

Faringoamigdalitis

aguda

Otitis Media Aguda

Laringitis Aguda

Bronquitis Aguda

Neumonia

Gráfica 2. Frecuencia de IRAS en menores de 5 años según diagnóstico clínico, C.S. Río Blanco, 2017. Mayor frecuencia de Faringoamigdalitis aguda, seguido del resfrío común. Fuente: PROPIA (Autor cubierto por doble ciego)

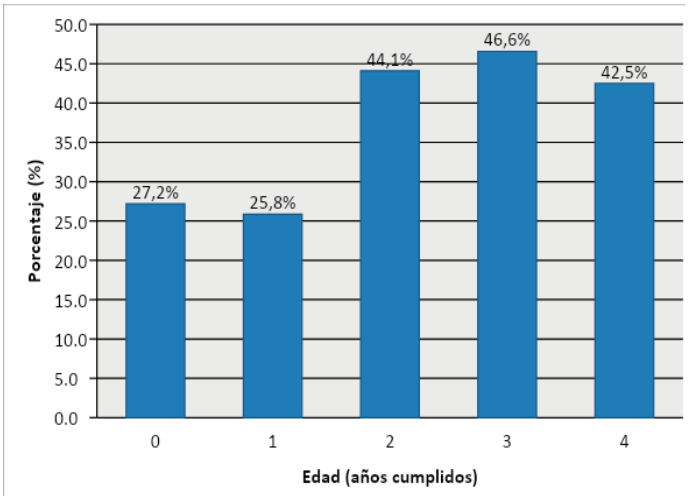

Gráfica 3. Frecuencia de IRAS en menores de 5 años según edad, C.S. Río Blanco, 2017. Mayor frecuencia de IRAS en edades $>2$ años ( $\mathrm{N}=3$ 510). Fuente: PROPIA (Autor cubierto por doble ciego)

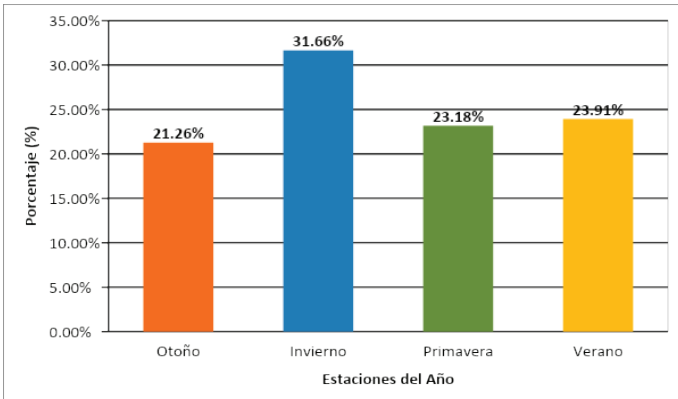

Gráfica 4. Frecuencia de IRAS en menores de 5 años según estaciones del año, C.S. Rio Blanco, 2017. La tercera parte de las IRAS en menores de 5 años se registraron en invierno (N: 1096). Fuente: PROPIA (Autor cubierto por doble ciego)

Tabla 1. Leve predominio de IRAS en el género femenino, no significativo.

Frecuencia de IRAS en menores de 5 años según género en el C.S. Rio Blanco, gestión 2017

\begin{tabular}{|ccc|}
\hline Genero & $\mathbf{N}^{\circ}$ & $\%$ \\
\hline Masculino & 535 & $48,81 \%$ \\
\hline Femenino & 561 & $51,19 \%$ \\
\hline Total & 1096 & $100 \%$ \\
\hline
\end{tabular}

FUENTE: PROPIA (Autor cubierto por doble ciego)

\section{DISCUSIÓN}

Tras identificar una frecuencia de IRAS del $31 \%$ en los menores de 5 años atendidos en el C.S. Río Blanco se evidencia la importancia de este grupo de enfermedades en la población que acude a dicho centro de salud puesto que prácticamente la tercera parte de la población de este grupo acude con problemas respiratorios de causa infecciosa, resulta un 
dato concordante con lo esperado para una comunidad del área rural de un país en vías de desarrollo como es Bolivia². No obstante, resaltar que la cifra identificada en este estudio es menor a la esperada según los datos del INE a nivel regional y nacional (31\% contra $34.3 \%$ y $41,6 \%$, respectivamente) ${ }^{6}$. En el contraste con otros estudios se observa que los datos obtenidos en esta investigación son similares a los encontrados por Córdova-Sotomayor DA, et al $^{7}$ en un Centro Materno-Infantil Santa Anita de Lima, Perú y son parcialmente más altos que los obtenidos por Valencia-Henao DC, et $a^{8}$ en el Centro de Salud Terrón Colorado de Cali, Colombia ( $30.5 \%$ y $26,6 \%$ de frecuencia de IRAS, respectivamente).

En este estudio se determinó como diagnóstico más frecuente la faringoamigdalitis aguda con $44 \%$ de frecuencia, en contraste con el estudio de Orellana-Cobos D, et al ${ }^{1}$, realizado en el Centro de Salud Cuchil de Ecuador en el que el resfrío común o rinofaringitis aguda fue la más frecuente con $69 \%$, sin embargo dichos resultados no se alejan tanto de nuestro estudio puesto que el resfrío común representa el segundo mayor diagnóstico con 37\%. En un intento de analizar esta diferencia de cifras proponemos que el factor desnutrición, asociado en el trabajo de Orellana-Cobos D, et al, cumple un papel muy importante. También se encuentran diferencias con el estudio de Chimborazo-Jarama $\mathrm{LE}^{9}$ que identificó al resfrió común como la mayor causa de IRA en su estudio realizado en la comunidad de Racar, Ecuador, abordando la frecuencia de IRAS en una comunidad donde la presencia de humo y polvo por ladrilleras circundantes podría explicar muchos de sus hallazgos. Comparado con el estudio de Juy-Aguirre $\mathrm{E}$, el $\mathrm{al}^{10}$, realizado en el Hospital Distrital de Sayaxché al suroeste de Guatemala, se encuentran diferencias puesto que en este las neumonías y bronconeumonías resultan ser más frecuentes con un $93 \%$. Sin embargo no se especifican las características de atención de dicho hospital razón por la cual resulta dificultosa la búsqueda de alguna variable que explique las diferencias entre las cifras con respecto a otros hallazgos.

Al analizar la frecuencia según el género se observó leve predominio del sexo femenino (51\%) sobre el sexo masculino (49\%) no obstante ésta diferencia no se considera significativa y podría enunciarse que ambos sexos son afectados de igual manera; en contraste con lo mencionado en la literatura en el que el sexo masculino se ve más comprometido, pero resaltando, no de forma significativa. Al revisar otros trabajos publicados se obtiene cifras semejantes a nuestros hallazgos como en el trabajo de Martin-Rodríguez et $\mathrm{al}^{4}$ realizado en el policlínico Luis E. de la Paz en Cuba, con $56,60 \%$ de frecuencia en el sexo femenino y $43,40 \%$ en el sexo masculino. Sin embargo, la mayor parte de la literatura consultada hace referencia a un leve predominio en el sexo masculino como en el trabajo de OrellanaCobos et al ${ }^{1}$ realizado en Ecuador, Juy-Aguirre et $\mathrm{al}^{10}$ de Guatemala y Duran y Tribiño ${ }^{11}$ realizado en ESE Hospital Nuestra Señora de Fátima en Colombia con cifras de $52,14 \%, 55,70 \%$ y $54 \%$, respectivamente.

De igual forma se realizó el análisis de la frecuencia según edad y como se indica en la Grafica 3 se ha evidenciado mayores frecuencias de IRAS en niños y niñas de 2, 3 y 4 años cumplidos a diferencia de las edades de 0 en adelante y 1 año cumplido. Este es un hallazgo discordante con lo que afirma Kraune-Criales $\mathrm{V}^{2}$ que indica que la mayor frecuencia de IRAS se registra en menores de 2 años y discordante también con los resultados de Vargas-Velázquez $\mathrm{JMA}^{12}$, y Ferreira $\mathrm{E}$, et $\mathrm{al}^{13}$, quienes determinaron mayor frecuencia de infecciones respiratoria en niños de 0 a 11 meses. Varios trabajos respaldan a estos autores y en sus trabajos reafirman la mayor morbilidad y mortalidad en menores de 1 año como lo hacen LópezMilián MM, et al ${ }^{14}$ y Coronel-Carvajal C, et al ${ }^{15}$ en sus artículos, relacionando esta morbimortalidad con factores individuales propios de la edad, como, nacimiento prematuro, bajo peso al nacer, inmadurez inmunológica, hipogammaglobulinemia transitoria al disminuir los anticuerpos que son traspasados de la madre vía transplacentaria y el destete temprano que priva de todos los factores nutricionales e inmunológicos naturales de la madre al lactante. También se han observado factores socioculturales variables que no serán analizados en este documento. No obstante, el hecho de registrar la mayor frecuencia de IRAS en edades avanzadas estima una mejor respuesta fisiológica del individuo a la enfermedad.

Finalmente, se ha estudiado la frecuencia de IRAS según las estaciones de año, tratando 
de identificar los meses y condiciones donde más se presentarían estas patologías. Como se evidencia en la Grafica 4, la tercera parte de las IRAS atendidas en el C.S. Río Blanco se observan en invierno $(31,66 \%)$, seguido de las 3 estaciones restantes (verano, primavera, otoño) con similares proporciones; estos datos son concordantes con la literatura, donde se estima que la mayoría de los casos de infecciones respiratorias ocurren en invierno ${ }^{2}$, cuando las variaciones en el clima y temperaturas más bajas condicionarían el aumento de IRAS como lo menciona Córdova-Sotomayor DA, et $\mathrm{al}^{7}$ en su artículo.

\section{CONCLUSIÓN}

La frecuencia de IRAS en menores de 5 años atendidos en el C.S. Rio Blanco desde enero a diciembre del 2017 es del 31\%, lo que las convierte en la tercera causa de atención médica en este grupo de pacientes. Se reconoce a la faringoamigdalitis aguda como el diagnóstico más frecuente. Según el género se establece un leve predominio de las infecciones respiratorias en el sexo femenino que no se considera significativo; además según la edad las IRAS se presentan más en edades superiores a los 2 años, contrario a lo descrito en la literatura. En relación a las condiciones climatológicas la tercera parte de las IRAS se presentaron en los meses de invierno.

En general, la mayoría de los hallazgos encontrados son los esperados para las condiciones de la región en estudio de acuerdo a lo descrito en la literatura. 


\section{REFERENCIAS}

1.- Orellana D, Urgilez G, Larriva D, Fajardo P. Estudio Transversal: Prevalencia de Infecciones Respiratorias Agudas y su Asociación con Desnutrición en Pacientes Menores de 5 Años Atendidos en el Centro de Salud de Cuchil, 2016. Rev Med HJCA. 2017; 9(2): 170-75.

2.- Kaune-Criales V. INFECCIONES RESPIRATORIAS ALTAS. In Mazzi-Gonzales de Prada E, et al. Texto de la Catedra de Pediatria. Sexta ed. La Paz; 2013. 155-63.

3.- Rodríguez-Cañete G, Ledea-Capote $E$, Rojas-Fajardo A, Barban-Cordovi M. Acciones estratégicas sobre prevención de Infecciones Respiratorias Agudas en menores de cinco años. Multimed. 2016; 20(4): 78496.

4.- Martín-Rodríguez L, Delgado-Gutierrez G, Araujo-Rodriguez H, Hernandez-Lastres I, Figueredo-Mendieta R. Algunos factores de riesgo de infecciones respiratorias agudas en menores de 5 años. Mayo 20142015. Multimed. 2017; 21(2): 4-13.

5.- Gonzáles-Valdés J. Infecciones respiratorias en el niño. Revista Cubana de Pediatría. 2013; 85(2): 147-48.

6.- Unidad de Comunicacion y Difusion. INE - Instituto Nacional de Estadistica. [Online].; 2018 [cited 2016 Enero 15. Disponible en: https://www.ine.go6.6o/index. php/las-infecciones-respiratorias-agudas-afectan-a-416-de-[a-poblacion-infantil]

7.- Córdova-Sotomayor DA, Chávez-Bacilio CG, Bermejo-Vargas EW, Jara-Ccorahua XN, Santa Marĺa-Carlos FB. Prevalencia de infecciones respiratorias agudas en niños menores de 5 años en un centro materno-infantil de Lima. Horiz Med. 2020; 20(1): 54-60.

8.- Valencia-Henao DC, Pinzón- Gomez EM, Hernández-Carrillo M, Moran-Garreta LM, Santander-Palta DC, Gómez-Franco DC,
Aragón-GoveaRA. ENFERMEDAD RESPIRATORIA AGUDA EN MENORES DE 5 AÑOS ATENDIDOS EN UN CENTRO DE SALUD, CALI-COLOMBIA. Rev.Medica.Sanitas. 2017; 20 (2): 67-74.

9.- Chimborazo-Jarama L, Sánchez-Morocho C. PREVALENCIA DE INFECCIONES RESPIRATORIAS AGUDAS EN NIÑOS MENORES DE 5 AÑOS, RACAR 2017. Tesis. Cuenca: UNIVERSIDAD DE CUENCA, FACULTAD DE CIENCIAS MÉDICAS; 2018.

10.- Juy-Aguirre E, Céspedes-Floirian E, Rubal-Wong A, Maza-González A, Terán-Guardia C. Morbilidad por infecciones respiratorias agudas en menores de 5 años. MEDISAN. 2014; 18(11): 1490-8.

11.- Duran C, Tribiño Y. Infección respiratoria aguda en primera infancia, estudio de casos y controles, Suaza 2011. Revista Facultad de Salud. 2011; 3(2): 33-46.

12.- Vargas-Velázquez J, Escobar-Salinas J. PERFIL CLINICOY EPIDEMIOLOGICO DE LAS INFECCIONES RESPIRATORIAS EN MENORES DE 5 AÑOS INTERNADOS EN UN HOSPITAL DE PARAGUAY. ENERO 2012 A DICIEMBRE 2013. CIMEL. 2015; 20(2): 63-66.

13.- Ferreira $E$, Báez $R$, Trejo B, Ferreyra $L$, Delgado $G$, et a I. Infecciones respiratorias agudas en niños y signos de alarma identificados por padres y cuidadores en México. Salud pública de México. 2013; 55(2): 30713.

14.- López-Milián $M M$, Méndez-López $M$, Méndez-López M, Nicot-Garague A. Infecciones respiratorias agudas: breve recorrido que justifica su comportamiento. Rev Inf Cient. 2016; 95(2): 339-55.

15.- Coronel-Carvajal C, Huerta-Montaña Y, Ramos-Téllez O. Factores de riesgo de la infección respiratoria aguda en menores de cinco años. Rev. Arch Med Camagüey. 2018;22(2):194-203. 